03.5;06.4;09.5;14.2

\title{
Генерация затопленной струи при лазерном нагреве поверхности жидкости
}

\author{
(С) В.М. Чудновский ${ }^{1}$, В.И. Юсупов ${ }^{2}$ \\ ${ }^{1}$ Тихоокеанский океанологический институт им. В.И. Ильичева ДВО РАН, Владивосток, Россия \\ ${ }^{2}$ Институт фоотонных технологий ФНИЦ „Кристаллография и фотоника“ РАН, Москва, Троицк, Россия \\ E-mail:vm53@mail.ru
}

Поступило в Редакцию 17 июня 2020 г.

В окончательной редакции 17 июля 2020 г.

Принято к публикации 17 июля 2020 г.

\begin{abstract}
Экспериментально обнаружено, что при нагреве поверхности воды непрерывным лазерным излучением с длиной волны $1.94 \mu \mathrm{m}$ через оптоволокно генерируются струи: одна - затопленная, направленная в глубь жидкости, другая - направленная в противоположном направлении, вверх в атмосферу. Струи образуются при схлопывании газовой полости, возникающей в процессе взрывного вскипания воды, вызванного поглощением лазерного излучения непосредственно под торцом оптоволокна, направленного вертикально вниз к поверхности жидкости. Обсуждается механизм образования струй.
\end{abstract}

Ключевые слова: лазерное излучение, затопленная струя, взрывное кипение, полость.

DOI: 10.21883/PJTF.2020.20.50153.18426

Неравновесные пузырьки, когда форма их поверхности не является сферически-симметричной, при коллапсе в жидкости способны генерировать затопленные струи, которые, невзирая на малые размеры пузырьков и масштабы струй, локально могут создать гигантское гидродинамическое давление, что используется, например, для очистки поверхностей и трансмембранного переноса веществ в клеточных технологиях $[1,2]$. В связи с этим большой интерес представляют паровые и парогазовые пузырьки, возникающие при кипении в жидкости, температура которой ниже температуры насыщения („кипение с недогревом“, цит. по [3]). В период своего роста они достигают максимального размера и затем в схлопываются. В том случае, когда пузырьки оказываются сферически-несимметричными, может происходить образование затопленных струй, скорость которых достигает от нескольких сантиметров до нескольких десятков и сотен метров в секунду [4-9]. С использованием лазеров появилась возможность нагревать жидкость и генерировать неравновесные паровые пузырьки в новых безальтернативных ситуациях, а также в тех случаях, когда обычный электрический нагрев не может быть применен либо неудобен. Для этого используют как импульсное $[9,10]$, так и непрерывное $[4,11]$ лазерное излучение. Использование намного менее затратного и более безопасного непрерывного лазерного излучения [4] очень перспективно, поскольку позволяет проводить контролируемую и неразрушающую очистку и обработку поверхностей, в том числе биологических.

Цель настоящей работы - показать, что нагрев поверхности жидкости непрерывным лазерным излучением может приводить к генерации затопленной струи, направленной от поверхности в глубь жидкости, и одновременно струи, противоположно направленной в атмосферу.

Излучение от волоконного лазерного аппарата ЛС-1.9 (ИРЭ-Полюс, Россия) с длиной волны $\lambda=1.94 \mu \mathrm{m}$, которое очень сильно поглощается в воде с коэффициентом поглощения $\alpha=130.0 \pm 0.5 \mathrm{~cm}^{-1}$ [12], подводилось по оптоволокну с диаметром кварцевой жилы $400 \mu \mathrm{m}$ к поверхности воды, находящейся в прозрачной для видимого света кювете размером $2.5 \times 2.5 \times 7 \mathrm{~cm}$ при температуре $22^{\circ} \mathrm{C}$. Расстояние от торца оптоволокна до поверхности воды составляло $500 \mu \mathrm{m}$, мощность лазерного излучения $2 \mathrm{~W}$. Динамика жидкости вблизи поверхности исследовалась с помощью скоростной камеры Fastcam SA-3 (Photron, Япония) на просвет со скоростью $10000 \mathrm{fps}$. На рис. 1 показаны кадры скоростной съемки при вскипании тонкого слоя поверхности воды под действием лазерного излучения. Особенностью данного процесса является то, что в начальный период взрывным образом формируется и растет паровой пузырек, тогда как его коллапс (схлопывание) - это уже коллапс газового - воздушного - пузырька. Вскипание приводит к образованию на поверхности жидкости полости, которая заполняется втекающим из атмосферы воздухом.

На кадрах первоначально образованная паровая полость расширяется аксиально вглубь и радиально в стороны. Пар частично выбрасывается в атмосферу, образуя реактивную струю, создающую дополнительное давление в аксиальном направлении в глубь жидкости (рис. 2). Как только давление пара становится меньше атмосферного, в образовавшуюся полость втекает атмосферный газ, и полость становится газовой. Динамика полости определяется замыканием перемычки на поверхности пузырька. На поверхности жидкость через $1.5 \mathrm{~ms}$ ра- 

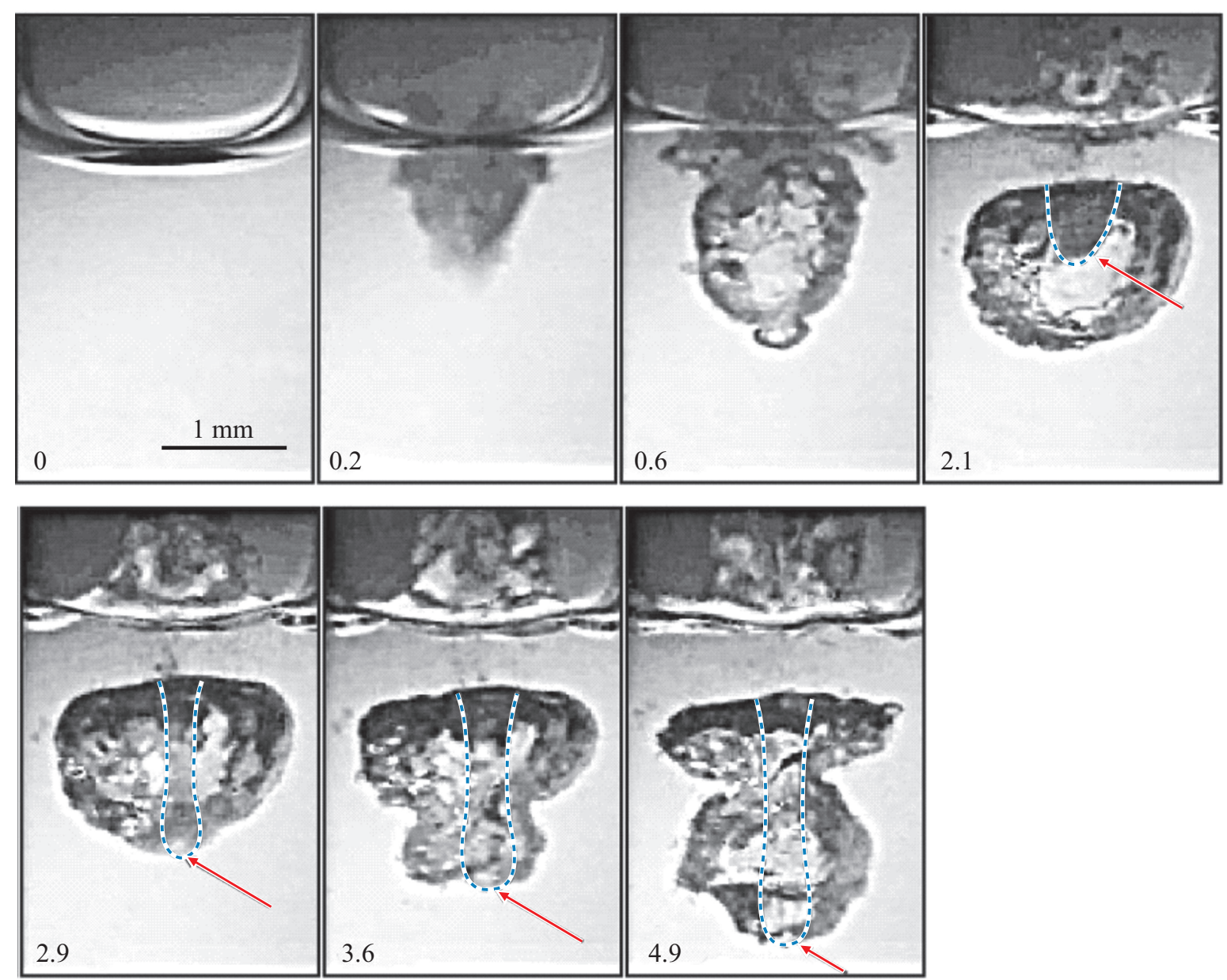

Рис. 1. Кадры скоростной съемки в момент образования затопленной струи при лазерном нагреве поверхности жидкости (видео доступно в онлайн-версии статьи). Время (в ms) отсчитывается от начала вскипания (кадр 0). Стрелкой показан фронт струи, пронизывающей пузырек.

диально схлопывается, что сопровождается возникновением аксиальных струй, одна из которых затопленная, распространяющаяся вглубь, а другая - распространяющаяся в противоположном направлении, вверх в атмосферу вдоль оптоволокна. Струя обнаруживается на кадрах, соответствующих 2.1-4.9 ms (рис. 1), внутри газового пузырька, который она в итоге полностью пересекает и выходит в жидкость в виде затопленной струи.

Модель, объясняющая образование затопленной струи, показана на рис. 2.

Вследствие сильного поглощения лазерного излучения на поверхности воды под торцом волокна образуется тонкий $(\sim 1 / \alpha \approx 80 \mu \mathrm{m})$ слой перегретой жидкости [12], перегрев в котором в принципе может быть сопоставим с величиной максимально достижимого в опытах перегрева в $210^{\circ} \mathrm{C}$ (см. работу [13] и ссылки в ней). В таком случае вскипание воды является бурным [14] и, как следствие, сопровождается выбросом в атмосферу пара с большой скоростью. При этом на жидкость действует импульс отдачи. В результате этих процессов в воде образуется пузырек в виде расширяющейся и быстро углубляющейся полости. Если предположить, что по выходу пара из полости, образованной вскипанием, атмосферный воздух устремляется в нее как в вакуум, то его скорость в горловине образовавшейся полости будет достигать $300 \mathrm{~m} / \mathrm{s}$. Это означает, что давление на уровне линии поверхности жидкости относительно атмосферного давления, согласно закону Бернулли, станет практически нулевым, что приведет к радиальному встречному движению жидкости. Максимальной эта скорость будет на линии поверхности жидкости. Именно там, как следует из рис. 1, происходит замыкание перемычки или горловины полости, заполненной воздухом. Время замыкания перемычки (горловины) $t_{\text {pinch }}$ газовой полости, соответствующей данной экспериментальной ситуации, определяется выражением [15]:

$$
t_{\text {pinch }}=C_{0} R_{0}\left(\rho R_{0} / \sigma\right)^{1 / 2}
$$

где константа $C_{0} \approx 0.874, R_{0}-$ начальный радиус полости в жидкости, заполненной воздухом к моменту начала ее коллапса на уровне поверхности жидкости, 


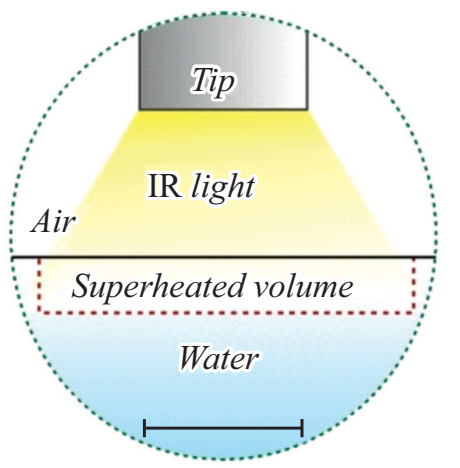

Heating

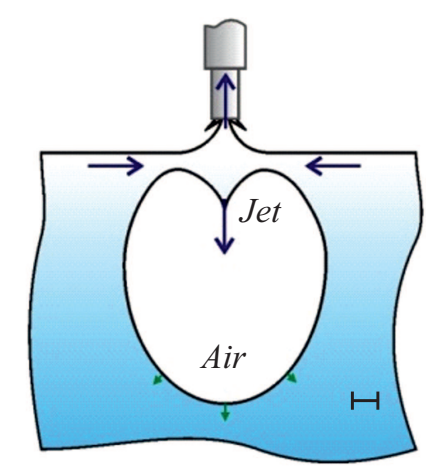

$\longrightarrow$ Jet and counter-jet

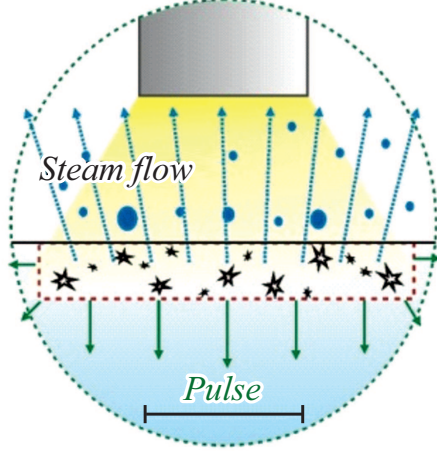

Explosive boiling

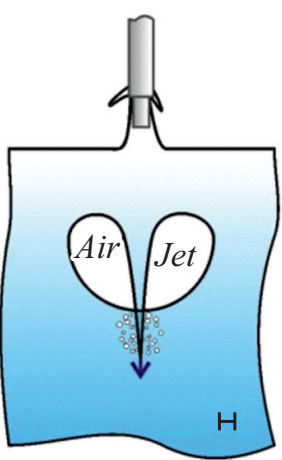

Wall breaking
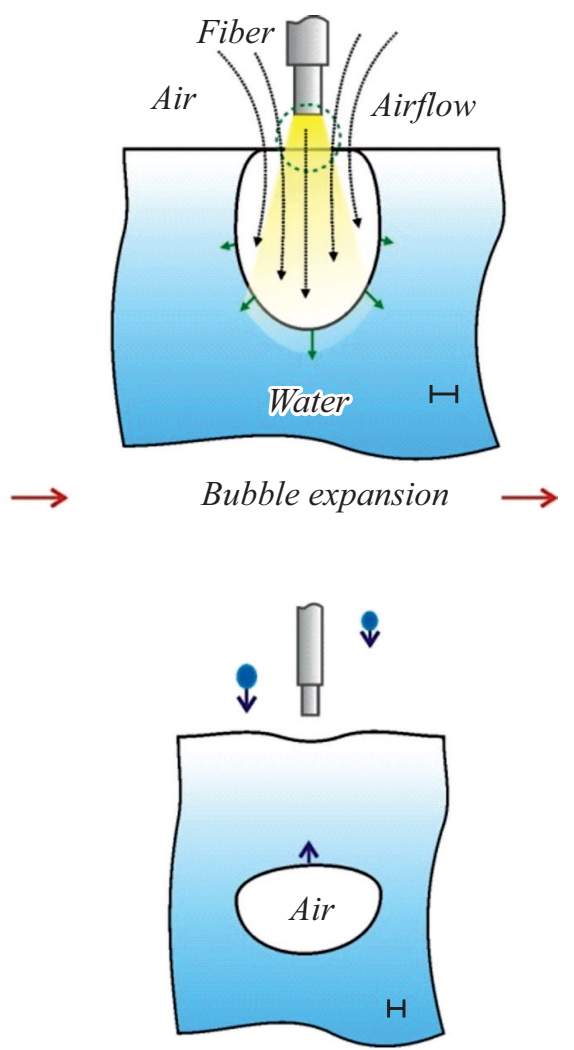

Bubble rising

Рис. 2. Модель образования струй при лазерном нагреве поверхности жидкости.

$\rho$ - плотность воды, $\sigma$ - коэффициент поверхностного натяжения воды, взятый при $100^{\circ} \mathrm{C}$. Подставляя исходя из рис. 1 значение $R_{0} \approx 1 \mathrm{~mm}$, получим $t_{\text {pinch }} \approx 3.6 \mathrm{~ms}$. Экспериментальное значение этого параметра соответствует кадрам с временем между $1.5-1.9 \mathrm{~ms}$. Таким образом, время замыкания перемычки, полученное экспериментально, приблизительно в 2 раза меньше рассчитанного теоретически, что можно объяснить исключением из рассмотрения эффектов от движения воздуха в полости и в данной ситуации считать вполне удовлетворительным соответствием. Это время позволяет определить среднюю скорость $V_{\text {mean }}$ встречного радиального движения жидкости на пути „лобового“ столкновения, приводящего к появлению аксиальных струй, направленных в противоположные стороны. Из эксперимента (рис. 1) находим $V_{\text {mean }} \approx 0.6 \mathrm{~m} / \mathrm{s}$, тогда как средняя скорость струи, пронизывающей воздушный пузырек, достигает $2 \mathrm{~m} / \mathrm{s}$. Гидродинамическое давление, создаваемое затопленной струей, движущейся со скоростью $2 \mathrm{~m} / \mathrm{s}$, составляет $P=\rho V^{2} / 2 \approx 2 \mathrm{kPa}$. Такое давление в затопленных струях позволяет с большой эффективностью очищать поверхности, погруженные в жидкость, не разрушая их. Последнее обстоятельство имеет решающее значение в медицине, поскольку позволяет атравматично санировать кожные покровы. Генерируемые подобным образом затопленные струи позволяют эффективно пе- реносить тепло, запасенное при лазерном нагреве, через объем холодной окружающей жидкости, что также имеет большое значение в хирургии.

\section{Финансирование работы}

Исследование выполнено при поддержке Министерства науки и высшего образования РФ в рамках выполнения работ по государственному заданию ФНИЦ „Кристаллография и фотоника“ РАН в части термокавитации, госбюджетной темы АААА-А20-120031890011-8 в части гидродинамики и гранта Российского фонда фундаментальных исследований № 18-02-00165 в части использования лазерных воздействий в медицине.

\section{Конфликт интересов}

Авторы заявляют, что у них нет конфликта интересов.

\section{Список литературы}

[1] Reuter F., Mettin R. // Ultrason. Sonochem. 2016. V. 29. P. 550-562.

[2] Dijkink R., Le Gac S., Nijhuis E., Van Den Berg A., Vermes I., Poot A., Ohl C.-D. // Phys. Med. Biol. 2008. V. 53. P. 375-390.

[3] Несис Е.И. Кипение жидкостей. М.: Наука, 1973. 280 с. 
[4] Chudnovskii V.M., Levin A.A., Yusupov V.I., Guzev M.A., Chernov A.A. // Int. J. Heat Mass Transfer. 2020. V. 150. P. 119286.

[5] Lu J.F., Peng X.F. // Int. J. Heat Mass Transfer. 2007. V. 50. P. 3966-3976.

[6] Zhukov S.A., Afanas'ev S.Yu., Echmaev S.B. // Int. J. Heat Mass Transfer. 2003. V. 46. P. 3411-3427.

[7] Li F., Gonzalez-Avila S.R., Nguyen D.M., Ohl C.-D. // Phys. Rev. Fluids. 2017. V. 2. P. 014007.

[8] Zhang A.M., Cui P., Wang Y. // Exp. Fluids. 2013. V. 54. P. 1602.

[9] Vogel A., Venugopalan V.// Chem. Rev. 2003. V. 103. P. 577644.

[10] Robles V., Gutierrez-Herrera E., Devia-Cruz L.F., Banks D., Camacho-Lopez S., Aguilar G. // Phys. Fluids. 2020. V. 32. P. 042005.

[11] Михеенко А.В. // Вестн. Тихоокеан. гос. ун-та. 2015. № 4 (39). C. 41-47.

[12] Deng R., He Y., Qin Y., Chen Q., Chen L. // Yaogan Xuebao - J. Remote Sensing. 2012. V. 16. P. 192-206.

[13] Rutin S.B., Skripov P.V. // Thermochim. Acta. 2013. V. 562. P. 70-74.

[14] Гурашкин А.Л., Старостин А.А., Скрипов П.В. // Письма в ЖТФ. 2020. Т. 46. В. 12. С. 47-50.

[15] Aristoff J.M., Bush J.W. // J. Fluid Mech. 2009. V. 619. P. 4578. 